\title{
LOCAL VARIABILITY IN THE ORBIT OF SATURN'S F RING
}

\author{
N. J. Cooper, C. D. Murray, and G. A. Williams \\ Astronomy Unit, School of Physics and Astronomy, Queen Mary University of London, Mile End Road, London E1 4NS, UK; n.cooper@qmul.ac.uk \\ Received 2012 October 22; accepted 2013 April 12; published 2013 May 13
}

\begin{abstract}
We present an analysis of the orbit of Saturn's F ring using images recorded by the Imaging Science Subsystem of the Cassini spacecraft. A total of 9805 observations have been made from 10 image sequences obtained between 2006 November 23 and 2009 July 28. Each sequence of up to 240 images spans a single orbit of the F ring, allowing 10 independent high-precision estimates of the ring orbit to be made over this $\sim 3$ year period. The ring has been modeled as an inclined uniformly precessing ellipse. The results show a variability in the orbital elements with, for example, the semi-major axis scattered between 140211.2 $\pm 0.1 \mathrm{~km}$ and $140232.9 \pm 0.4 \mathrm{~km}$ and the fitted periapses locked to the value obtained from a combined fit using the entire three-year span of observations. We show that the observed scatter between the individual estimates of the ring orbit reflect the differing past histories of the particular segments of ring being fitted and that the values are scattered within the limits expected from a single gravitational encounter with the nearby moon, Prometheus. In the combined fit, the scatter averages out to reveal a small systematic bias with respect to the results of Bosh et al. and Albers et al. We believe this is a consequence of the proximity of Prometheus to the ring in the image sequences chosen for this analysis. Finally, we note a close empirical commensurability between the apsidal precession rate, $\dot{\varpi}$, of the $\mathrm{F}$ ring and the synodic period between Prometheus and the F ring, such that $n_{\text {Prom }}-n_{\text {Fring }} \approx 2 \dot{\omega}_{\text {Fring }}$, where $n_{\text {Prom }}$ and $n_{\text {Fring }}$ are the mean motions, and discuss its implications.
\end{abstract}

Key words: astrometry - planets and satellites: dynamical evolution and stability - planets and satellites: individual (Saturn) - planets and satellites: rings

Online-only material: color figures

\section{INTRODUCTION}

First detected optically by the Pioneer 11 spacecraft and then imaged more extensively by the Voyager spacecraft and the Imaging Science Subsystem (ISS) of the Cassini orbiter, the F ring of Saturn has a highly irregular visual appearance, which has required a whole new vocabulary to describe the variety and complexity of observed physical features: streamers and channels, spirals, jets and mini-jets, fans, and clumps. The underlying physical mechanisms responsible for many of these features are now well-understood: streamer/channel structures are produced by gravitational perturbations from Prometheus and to a lesser extent Pandora (Guiliatti Winter 1994; Murray et al. 2005); fans are caused by the perturbation of embedded objects, while jets, strands, spirals and mini-jets are formed by collisional processes (Charnoz et al 2005; Murray et al. 2008; Attree et al. 2012). However, the obvious visual complexity of the $\mathrm{F}$ ring region is in stark contrast with existing orbit models derived from stellar occultation observations, which describe a well-behaved Keplerian, uniformly precessing, inclined, elliptical ring (Bosh et al. 2002; Albers et al. 2012).

In this paper, we address this apparent contradiction. Our aim is not to provide a single "definitive" orbit for the F ring from Cassini ISS data. Instead, we demonstrate that it is possible to detect local variability in the orbit of the ring consistent with its irregular visual appearance while showing also that the longer-term behavior, averaged on a timescale of years, is largely consistent with stellar occultation results (Bosh et al. 2002; Albers et al. 2012). We argue that the notion of a "definitive orbit" for the ring has limited meaning in the context of this local variability, except to the extent that the ring appears to precess uniformly. Locally, we find a range of orbits reflecting the dynamical history of the particular segment of ring being observed, a history dominated in the images used in this study by the effects of gravitational perturbations due to Prometheus.
Our methodology involves using images from the Cassini ISS rather than stellar occultation observations. The particular benefit of the image sequences used in this study is that they have allowed the orbit of the ring to be modeled both in a local and an averaged sense. Specifically, each image sequence follows a $\sim 5^{\circ}$ segment of the $\mathrm{F}$ ring for almost one complete orbit, allowing a precise estimate of the orbit of that particular segment of the ring to be made. By comparing a number of these local orbits separated by several months, we have been able to evaluate possible time-variant changes in the orbit, and in addition to compare these local estimates with the averaged behavior of the orbit between 2006 November 23 and 2009 July 28 , the latter produced by combining the observations for all the image sequences. We then compare the local and averaged orbits with the estimates obtained from stellar occultation observations (Bosh et al. 2002; Albers et al. 2012), showing how the proximity of the nearby satellite Prometheus locally influences the orbit of the ring.

We emphasize that, unlike Showalter (2004), our method does not involve the tracking of specific embedded objects or ring features from image-to-image (generally there is nothing to track). Rather, we have performed a geometrical fit to points selected essentially randomly from raw images of the $\mathrm{F}$ ring core, avoiding obvious streamer/channel structures where possible. The positions of the measured points are not related from image to image in any deliberate way. In this limited respect, our approach has more in common with stellar occultation-based methods.

\section{OBSERVATIONS}

Since Saturn Orbit Insertion (SOI) in 2004, two complementary approaches have been developed to image the $\mathrm{F}$ ring using the Cassini ISS. In the first, so-called STREAMER/CHANNEL image sequences have been designed to observe the local effects 

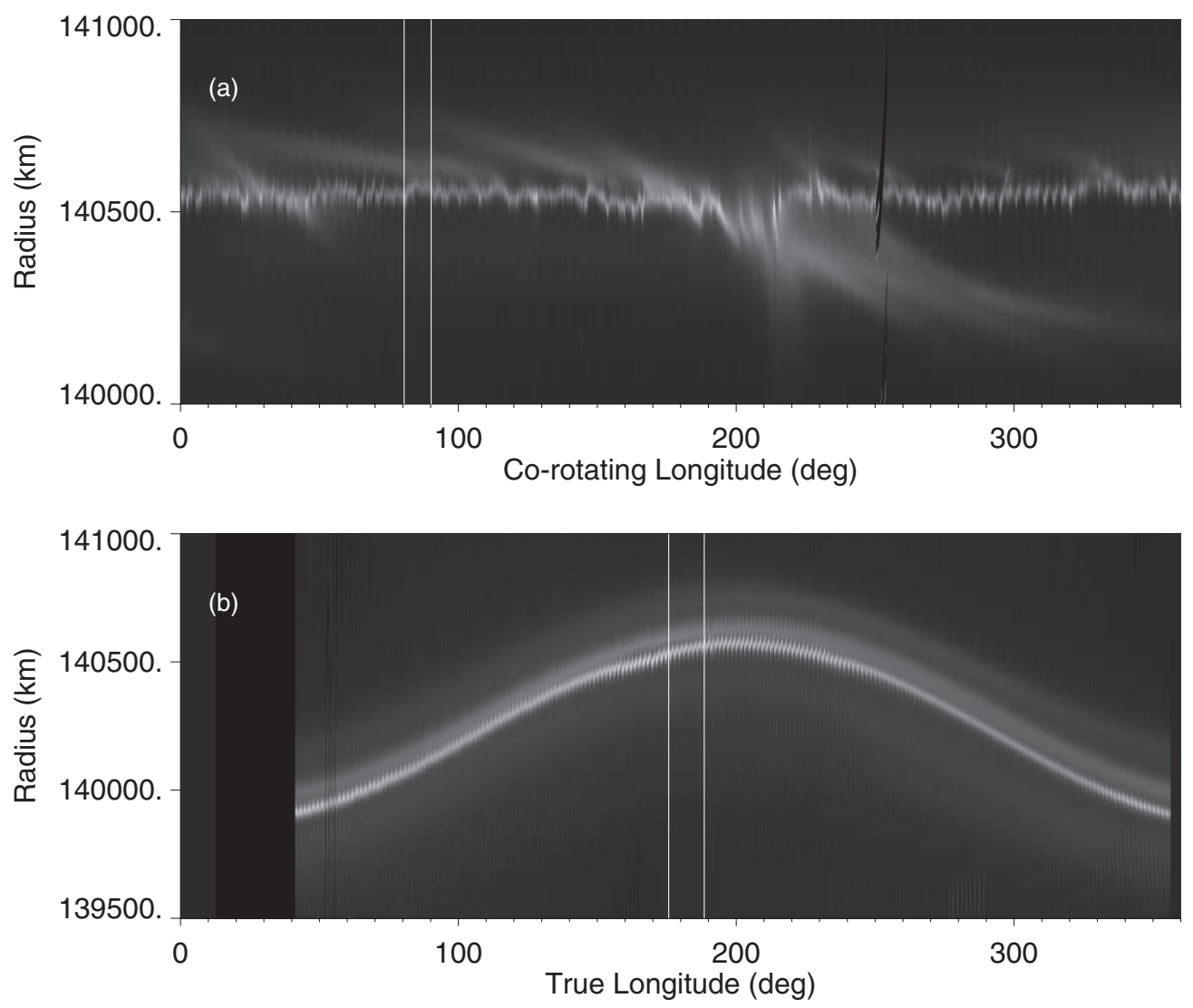

Figure 1. Comparison between (a) FMOVIE and (b) STREAMER/CHANNEL image sequences. Raw images have been reprojected into radial distance from Saturn vs. longitude then combined. Each row of pixels corresponds to a fixed radial distance from Saturn and each column to a fixed longitude. Reprojections are in the equatorial plane of Saturn. (a) FMOVIE sequence ISS_043RF_FMOVIE001_VIMS, covering $360^{\circ}$ in longitude co-rotating with the mean motion of the $F$ ring (using the value $581.96 \mathrm{deg} / \mathrm{day}$ ), with true longitude range of $175^{\circ} .7-188^{\circ} .5$ and observation date 2007 DOY 108 (b) STREAMER/CHANNEL sequence, ISS_043RF_FRSTRCHAN001_PRIME, covering $\sim 360^{\circ}$ of true longitude, with equivalent co-rotating longitude range from 80.5 to $90^{\circ} 2$ and observation date of 2007 DOY 110. The segment of F ring sampled by the STREAMER/CHANNEL sequence is marked by the vertical lines in the FMOVIE display (a). The field-of-view used in the FMOVIE sequence is marked by the vertical lines in the STREAMER/CHANNEL display (b).

of Prometheus on the ring, by using the narrow angle camera (NAC) to follow a $\sim 5^{\circ}$ portion of the ring around approximately one complete orbit, with Prometheus maintained in the center of the field-of-view for each image (Murray et al. 2005). In the second approach, "FMOVIE" sequences have been designed to image the full variability of the F ring by using the NAC to stare at a fixed true longitude until the entire ring has passed through the field-of-view once (Murray et al. 2008). Thus "FMOVIE" sequences provide $360^{\circ}$ coverage in longitude co-rotating with the mean angular velocity of the ring, while imaging only a few degrees of true longitude centered on the chosen camera pointing direction.

A comparison between the two types of image sequence is shown in Figure 1. In each case, images have been reprojected and combined to give radial distance from Saturn's center as a function of longitude in degrees. The "FMOVIE" in Figure 1(a) emphasizes the variability of ring features as they pass through the field of view, but the radius is approximately constant because the camera is pointing at a fixed inertial longitude throughout. In contrast, the STREAMER/CHANNEL sequence in Figure 1(b) emphasizes the geometry of the ring orbit, clearly showing its eccentricity and the locations of apoapse and periapse, while conversely the local variability seen in the FMOVIE is largely absent, because only one small segment of ring is being followed around its orbit.

Since in this work we are interested primarily in the geometry of the ring, measurements of the position of the F ring have been made from STREAMER/CHANNEL image sequences, such as Figure 1(b), where each sequence provides up to $360^{\circ}$ of true longitude coverage for a single orbit of the ring. The full series of image sequences used in this work is summarized in Table 1.

The imaged position of the F ring was measured astrometrically from the raw ISS images using the CaViaR software package, developed at Queen Mary University of London, incorporating the NASA SPICE software (Acton 1996). Image line and sample coordinates were measured by manually clicking with a mouse at discrete but irregularly spaced locations along the core of the F ring in each raw image. In this work, we define the core to be the continuous ring feature corresponding to the location of the brightest pixel, measured from a raw image. Figure 2 shows an example of a raw image with radial brightness profile (this profile is for illustrative purposes only: profiles were not explicitly used in the measurement process). Even in cases such as in Figure 2, where multiple strands of the ring are present, a unique bright pixel exists that can be followed continuously across the image, consistent with the adopted criterion used to locate the core. Figure 2 is somewhat atypical in that there are two adjacent pixels of very similar brightness, but again, as in all cases, the brighter of the two has been selected, following our chosen definition. Image exposure durations ranged from 1 to $1.5 \mathrm{~s}$, except for some $0.180 \mathrm{~s}$ exposures in sequence ISS_033RF_FRSTRCHAN001_PRIME (Table 1). Exposure durations were chosen to ensure that the $\mathrm{F}$ ring was never saturated in the images. The optical image 


\begin{tabular}{|c|c|c|c|c|c|c|c|c|c|c|}
\hline Sequence & $\begin{array}{c}\text { Phase }^{\mathrm{a}} \\
\text { (deg) }\end{array}$ & $\begin{array}{c}\text { Elevation }^{\mathrm{b}} \\
(\mathrm{deg})\end{array}$ & $\begin{array}{l}\text { Exposure } \\
\text { Duration } \\
(\mathrm{ms})\end{array}$ & $\begin{array}{l}\text { Range of Radial } \\
\text { Resolution } \\
\left(\mathrm{km} \mathrm{pixel}^{-1}\right)\end{array}$ & $\begin{array}{c}\text { Range of Azimuthal } \\
\text { Resolution } \\
\left(\mathrm{km} \mathrm{pixel}^{-1}\right)\end{array}$ & $\begin{array}{l}\text { Min. True } \\
\text { Longitude }^{\mathrm{c}} \\
\quad \text { (deg) }\end{array}$ & $\begin{array}{l}\text { Max. True } \\
\text { Longitude }^{\mathrm{c}} \\
\quad \text { (deg) }\end{array}$ & $\begin{array}{l}\text { Min. Co-rotating } \\
\text { Longitude }^{\mathrm{d}} \\
\text { (deg) }\end{array}$ & $\begin{array}{l}\text { Max. Co-rotating } \\
\text { Longitude }^{\mathrm{d}} \\
\text { (deg) }\end{array}$ & $\begin{array}{c}\text { No. of } \\
\text { Images } \\
\text { Used }\end{array}$ \\
\hline ISS_033RF_FRSTRCHAN001_PRIME & 151.5 & 42.3 & $180,1200^{\mathrm{e}}$ & $8.5-13.9$ & $8.0-13.7$ & 2.66032 & 357.57777 & 15.45171 & 23.76041 & 97 \\
\hline ISS_043RF_FRSTRCHAN001_PRIME & 95.6 & 42.8 & 1200 & $10.2-18.0$ & $9.8-15.6$ & 0.00892 & 359.99997 & 79.93254 & 90.86521 & 210 \\
\hline ISS_044RF_FRSTRCHAN001_PRIME & 84.0 & 31.9 & 1000 & $10.9-25.0$ & $10.4-21.4$ & 0.00507 & 359.99612 & 162.85676 & 177.88784 & 240 \\
\hline ISS_080RF_FRSTRCHAN001_PRIME & 31.4 & 23.9 & 1200 & $6.7-24.2$ & $5.9-17.3$ & 0.00581 & 359.99690 & 98.93947 & 108.20182 & 214 \\
\hline ISS_088RF_FRSTRCHAN001_PRIME & 28.4 & 20.2 & 1000 & $6.8-26.3$ & $6.3-18.5$ & 0.82844 & 358.97408 & 51.49379 & 61.74980 & 197 \\
\hline ISS_091RF_FRSTRCHAN001_PRIME & 30.9 & 21.6 & 1200 & $6.6-23.9$ & $5.8-21.7$ & 1.28058 & 359.11040 & 166.69959 & 179.17346 & 197 \\
\hline ISS_102RF_FRSTRCHAN001_PRIME & 38.9 & 33.8 & 1500 & $6.1-9.9$ & $5.6-13.0$ & 68.34121 & 343.76614 & 306.35999 & 314.85502 & 160 \\
\hline ISS_103RF_FRSTRCHAN001_PRIME & 51.7 & 48.4 & 1500 & $6.6-11.8$ & $6.2-9.3$ & 17.81586 & 288.79802 & 2.81477 & 9.70247 & 209 \\
\hline ISS_109RF_FRSTRCHAN001_PRIME & 71.6 & -44.2 & 1200 & $6.0-11.7$ & $5.6-8.8$ & 1.21640 & 359.46947 & 36.34503 & 42.03686 & 206 \\
\hline ISS_115RF_FRSTRCHAN001_PRIME & 85.6 & 30.8 & 1000 & $7.8-18.9$ & $7.4-16.7$ & 0.19553 & 358.73902 & 182.43308 & 191.41745 & 146 \\
\hline
\end{tabular}

Notes.

${ }^{a}$ Mean spacecraft-ring-solar angle.

${ }^{\mathrm{b}}$ Mean angle between spacecraft-ring pointing vector and ring plane. A negative number indicates spacecraft below ring plane.

c True longitudes calculated relative to the ascending node of Saturn's equator on the ICRF equator.

${ }^{\mathrm{d}}$ Co-rotating longitudes calculated relative to epoch 2007 Jan 1 12:00:00.0 UTC using a mean motion of 581.96 deg day ${ }^{-1}$.

${ }^{\mathrm{e}}$ Approximately one image in five has $180 \mathrm{~ms}$ exposure duration. 


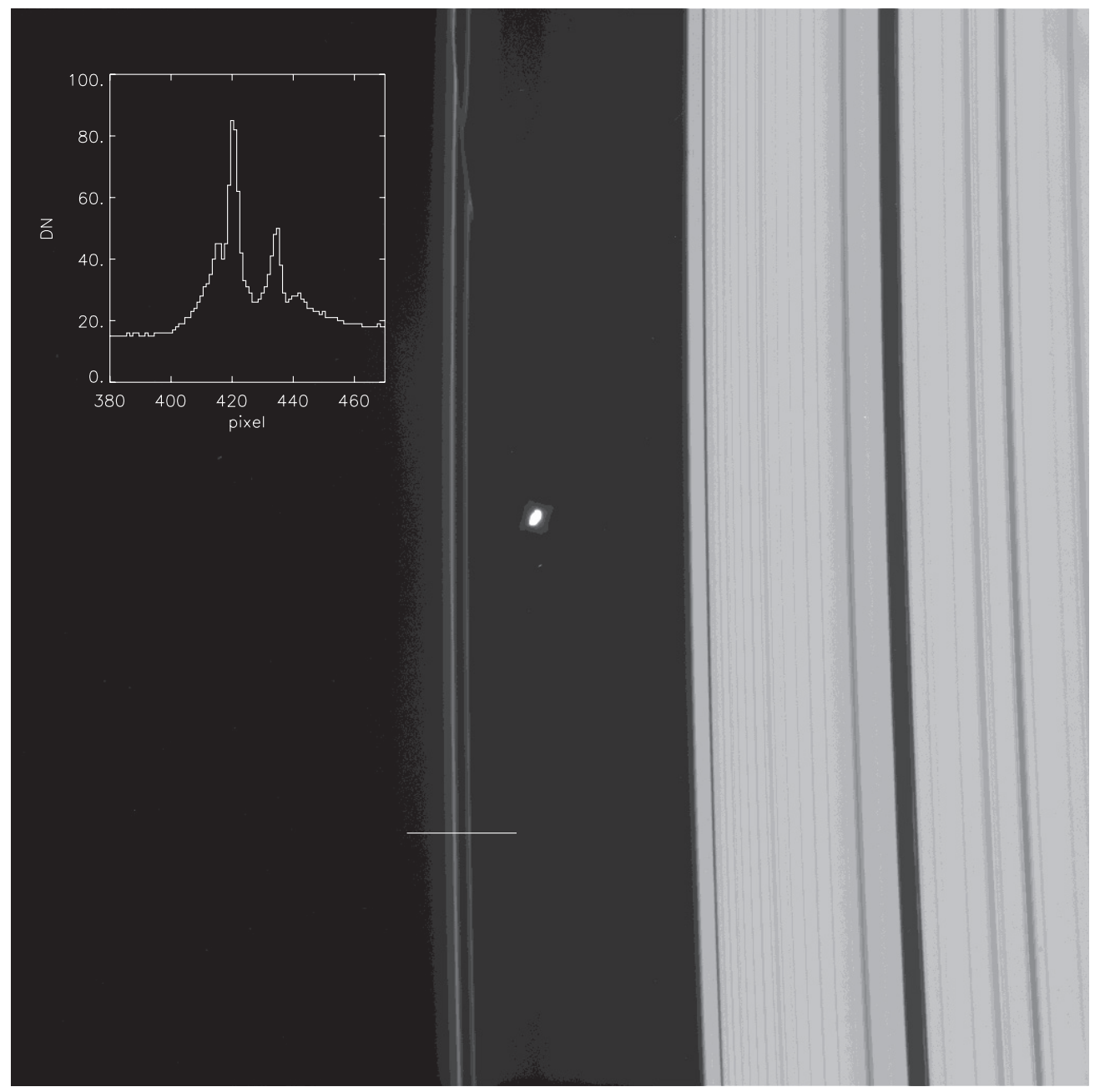

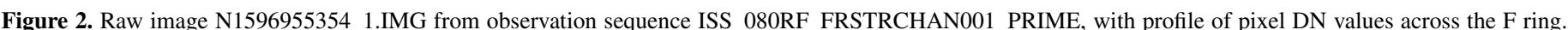

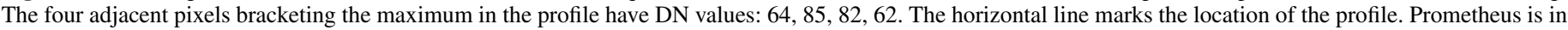

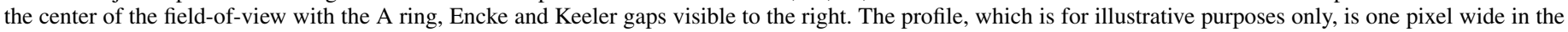
longitudinal direction.

sequences used in this work have the advantage over stellar occultation observations in that the continuity of the feature of interest can easily be followed visually, both within a given image and from one image to the next. In contrast, stellar occultations, often at time intervals of perhaps months, make consistent correlation of the core between discrete profiles potentially more difficult, especially given the structural complexity and dynamical nature of the $\mathrm{F}$ ring.

Typically five irregularly spaced measurements along the ring were made per image. Since each image covers on average $\sim 4.8$ in true longitude, this provides about one measurement every $\sim 1^{\circ}$ in longitude along the core. Taking into account the overlap in true longitude between adjacent images and the relative drift between the ring and Prometheus of about 2.5 over an $11 \mathrm{hr}$ sequence, this finally results on average, in $\sim 1000$ measurements spanning the $\sim 360^{\circ}$ of true longitude covered by each image sequence. In practice, there are gaps in the longitudinal coverage, caused by Saturn and its shadow, so full $360^{\circ}$ coverage is never quite achieved. However such fine longitudinal sampling is perhaps five orders of magnitude more dense than for a typical sequence of stellar occultation observations and is sufficient to provide a precise estimate of the orbital parameters corresponding to a single orbit of the $\mathrm{F}$ ring. In contrast, the time interval between individual image sequences (typically several months) is of the same order of magnitude as for typical stellar occultation observations, so when considering an orbit model fitted using all the imaging observations together, each sequence is in essence a single highprecision point. However, with 10 image sequences, we have only 10 such high precision points, in contrast to the $\sim 100$ used in comparable stellar occultation studies (Albers et al. 2012), and in even greater contrast to the $\sim 1000$ points measured within each individual sequence in this current work. Also, the radial resolution of the images is approximately $10 \mathrm{~km} \mathrm{pixel}^{-1}$, which is four orders of magnitude coarser than the "meter" resolution possible with stellar occultations. So, in summary, each observation method has its benefits, and the imaging and occultation methods can be considered to be complementary.

We experimented with automatic measurement of the core; however, it proved difficult to maintain a consistent fix on the core through disturbed areas, often causing "cycle skips" on to adjacent strands. The manual approach described above avoided such difficulties, since areas of the ring close to streamer/ channel structures, and other localized features where the core showed evidence of radial distortion, could simply be bypassed. This is an imperfect process though and some contamination 


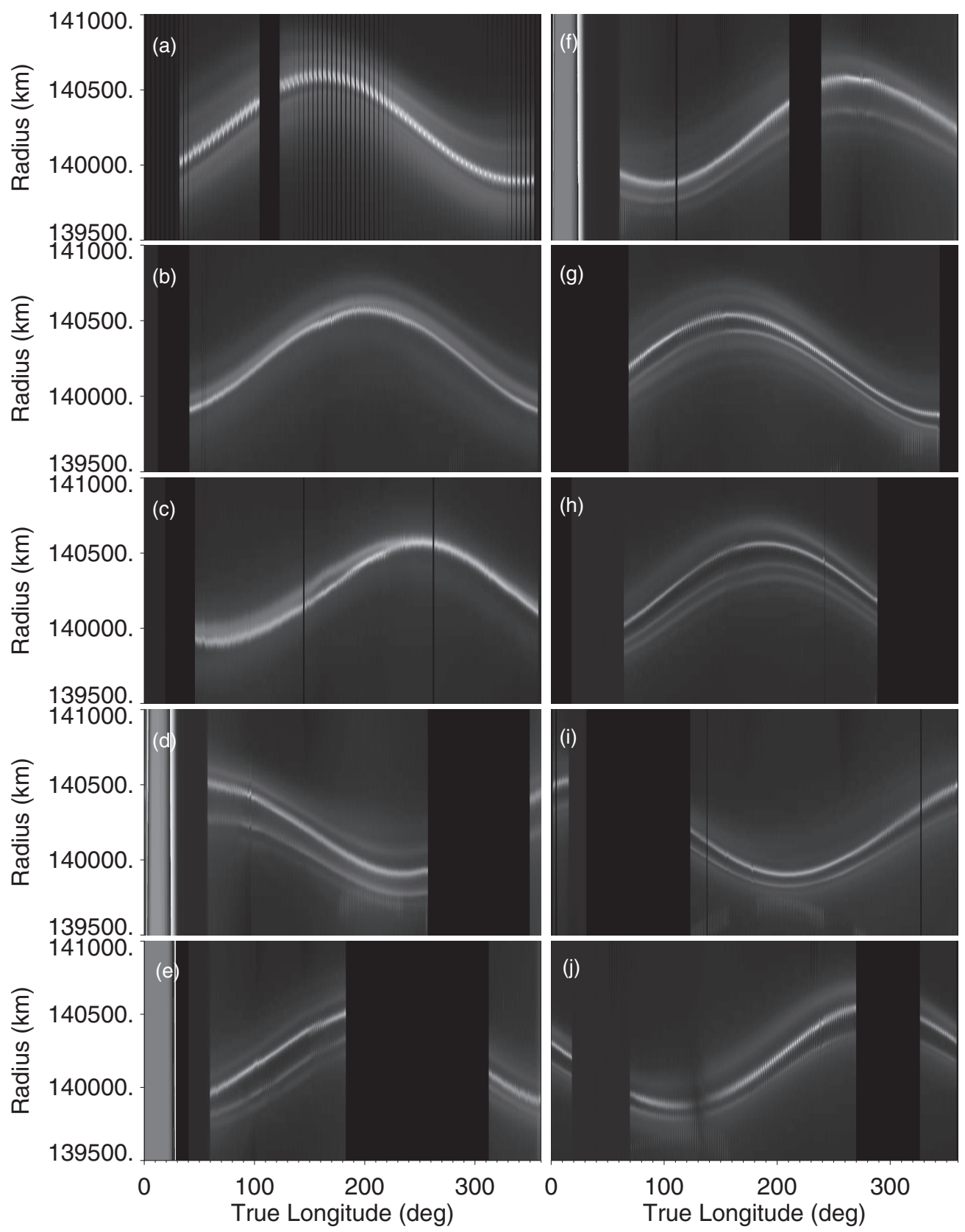

Figure 3. The complete set of reprojected STREAMER/CHANNEL mosaics for sequences ISS_revRF_FRSTRCHAN001_PRIME, where the rev numbers are given below. Each horizontal row of pixels corresponds to a fixed radial distance from Saturn. Each vertical column corresponds to a fixed true longitude. Reprojections are in the equatorial plane of Saturn. The sequence rev numbers, in increasing chronological order, are (a) 033, (b) 043, (c) 044, (d) 080, (e) 088, (f) 091, (g) 102, (h) 103, (i) 109, and (j) 115. See Table 3 for the dates corresponding to each sequence.

is inevitable. However this was mitigated by the large number of points generated for each observation sequence so that overall, any measurements from distorted areas contributed to the observed-minus-computed $(O-C)$ residuals following the orbit fitting (Table 3 and Figures 4-7). In addition, as we show subsequently, the effects of the most recent Prometheus perturbation can be controlled in the orbital analysis, by considering the angular separation between Prometheus and a given point on the ring.

In each NAC image, the nominal camera pointing direction was corrected using an iterative technique to match the calculated locations of background reference stars to their measured centroid positions, solving for the right ascension, declination and twist angle of the camera optical axis, corresponding to the center of the image. In this work, reference star positions were obtained from the UCAC2 and Tycho-2 star catalogs (Zacharias et al. 2004; Høg et al. 2000). The achieved pointing accuracy was $\sim 0.1$ NAC pixel $(0.12357$ arcsec $)$.

Measurements were made from a total of 10 separate STREAMER/CHANNEL image sequences. Reprojected mosaics for each of these are shown in Figure 3. In these displays, each horizontal row of pixels corresponds to a fixed radial distance from the center of Saturn, while the horizontal axis is true longitude. The eccentricity of the ring is immediately apparent, underlining just how useful these image sequences are for measuring the orbit of the ring, even though they were not specifically designed for that purpose. However, we emphasize once more that the astrometric measurements of the position of 


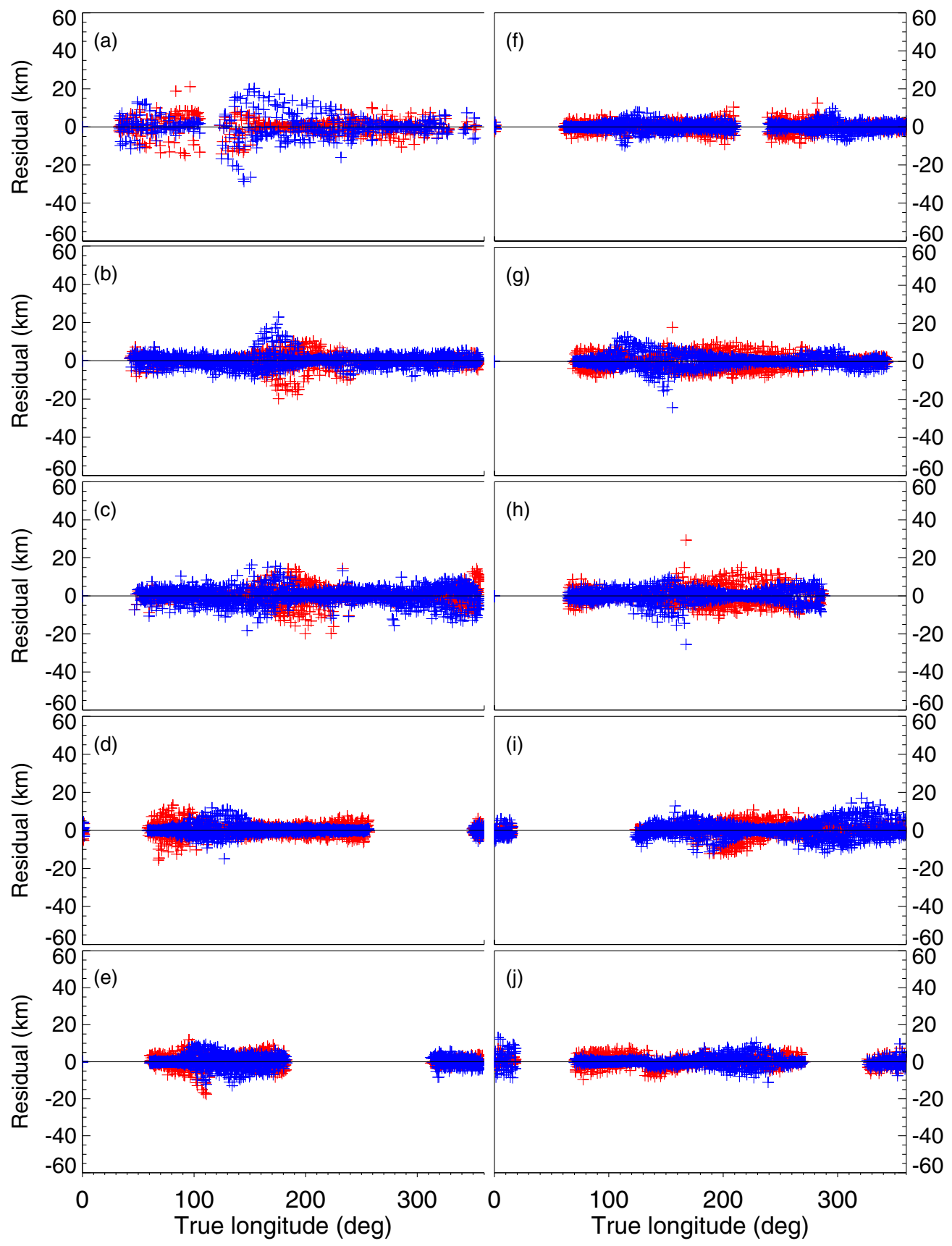

Figure 4. Residuals $(O-C)$ for fits $1-10$ in Table 3 as a function of true longitude (same horizontal scale as Figure 3). Line residuals (red) and sample residuals (blue) have been converted to units of km using the mean resolution for each image sequence.

(A color version of this figure is available in the online journal.)

the ring used in this study were made from the raw images, not from the reprojected mosaics shown in Figure 3, thus allowing a full three-dimensional orbit model to be used.

\section{ORBITAL SOLUTIONS}

The observations were modeled as a uniformly precessing, inclined, elliptical ring, with the following fitted parameters: semi-major axis, $a$, inclination, $i$, eccentricity, $e$, longitude of periapse, $\varpi$, and longitude of ascending node, $\Omega$. Where possible, the apsidal precession rate, $\dot{\varpi}$, was also fitted.

A planetocentric reference frame was adopted, with $x$-axis corresponding to the position of the ascending node of Saturn's equatorial plane on the mean Earth equator at the J2000 epoch
(2451545.0 JED). The $z$-axis is directed along Saturn's spin axis at epoch (pointing north) and the $y$-axis is orthogonal to $x$ and $z$. The key constants used in the orbit determination are given in Table 2. The Saturn pole position given in Table 2 was precessed from 1980 Nov 12 23:47:23.0 UTC to each individual fit epoch using rates of -0.04229 deg century $^{-1}$ in right ascension (R.A.) and -0.00444 deg century $^{-1}$ in declination (decl.; Jacobson 2004).

For each observation sequence, position vectors in inertial space at each observation time were calculated using an initial estimate of the orbital elements for the F ring core, based on the existing published models of Bosh et al. (2002) and Albers et al. (2012). The computed position vectors were then converted to equivalent image line and sample, using the corrected camera 

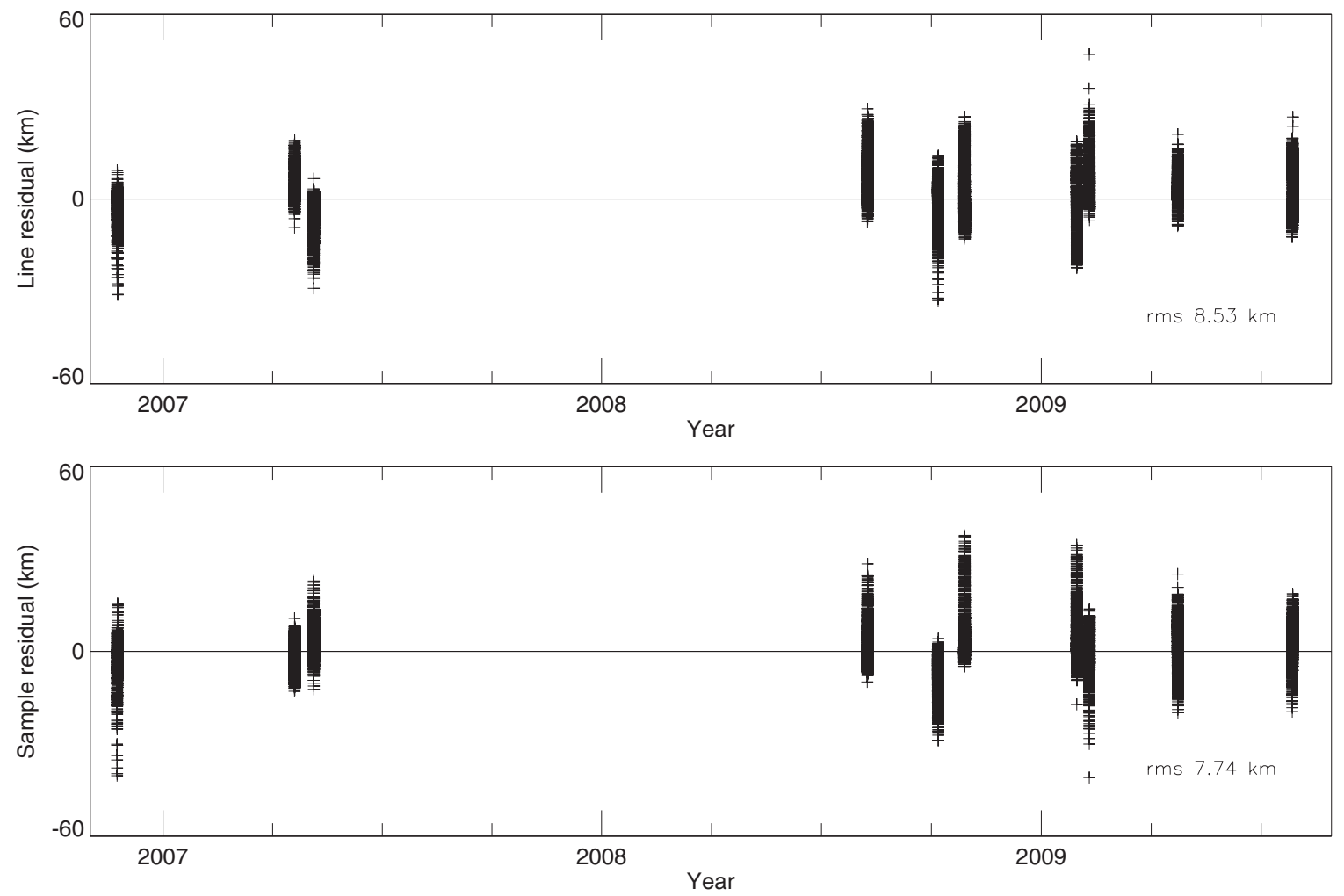

Figure 5. Residuals $(O-C)$ for the combined ISS fit 11 in Table 3 using observations from all 10 image sequences, plotted against time. Line residuals (upper display) and sample residuals (lower display) have been converted to units of km using the mean overall image resolution of $7.90 \mathrm{~km} \mathrm{pixel}^{-1}$.

Table 2

Saturn Constants Used in Orbit Determination

\begin{tabular}{lcc}
\hline \hline Constant & Value $^{\mathrm{a}}$ & Units \\
\hline Pole (R.A., decl.) & $(40.59550,83.53812)$ & $\mathrm{deg}$ \\
Pole epoch & $1980 \mathrm{NOV} \mathrm{12} \mathrm{23:47:23.0}$ & \\
Pole precession rate (R.A.) & -0.04229 & deg century \\
Pole precession rate (decl.) & -0.00444 & deg century $^{-1}$ \\
$G M$ & 37931284.0 & $\mathrm{~km}^{3} \mathrm{~s}^{-2}$ \\
Radius, $R_{S}$ & 60330 & $\mathrm{~km}$ \\
$J_{2}$ & 0.016292 & \\
$J_{4}$ & -0.000931 & \\
$J_{6}$ & 0.000091 & \\
\hline
\end{tabular}

Note. ${ }^{a}$ Pole position from Jacobson (2004). This position was precessed to each individual fit epoch. Reference radius from Kliore et al. (1980). Zonal harmonics and $G M$ from Jacobson (2004).

pointing direction and the equations of condition solved using an iterative least-squares procedure incorporating the SVD-based inversion method of Lawson \& Hanson (1975), minimizing the $O-C$ residuals in line and sample.

The quoted uncertainties in the orbital elements are the formal $1 \sigma$ values from the least-squares fitting. Factors affecting these uncertainties include the uncertainty in each measured core position, which we estimate to be $\sim 1$ pixel ( $\sim 8 \mathrm{~km}$ on average), the uncertainty in Saturn's pole position, estimated to be $\sim 1 \mathrm{~km}$ (Albers et al. 2012), and the pointing uncertainty of $\sim 0.1$ NAC pixel. Observations were uniformly weighted, so variability in image resolution was not explicitly taken into account and contributes to the overall rms error for each solution, along with the other factors mentioned.

Solutions are listed in Table 3. Fits 1-10 represent separate solutions for each of the 10 STREAMER/CHANNEL image sequences. For these solutions, fitted parameters are referenced to local time epochs, as given in the table, corresponding approximately to the time of the first observation for each sequence.

Solutions 11 and 12 (which we refer to in the text as "combined fits") represent fits to all 10 image sequences combined (a total of 9805 line/sample pairs in the case of fit 11), spanning the time range 2006 November to 2009 July. These solutions are referenced to the J2000 epoch, for direct comparison with existing published models of the F ring orbit derived from stellar occultation observations by Albers et al. (2012) and Bosh et al. (2002). The latter two previously published solutions are also provided in Table 3 for easy reference (fits 13 and 14, respectively). Fit 12 uses a subset of the ISS observations over the full time range, excluding those parts of the ring which have most recently encountered Prometheus. We discuss this solution further in the next section.

Comparing the 10 individual fits, $1-10$, the $O-C \mathrm{rms}$ residual varies between 2.3 and $5.5 \mathrm{~km}$, while for combined fit 11, an rms of $8.2 \mathrm{~km}$ was obtained. The latter compares to a value of $24.0 \mathrm{~km}$ obtained by Albers et al. (2012) and $5.7 \mathrm{~km}$ obtained by Bosh et al. (2002). $O-C$ fit residuals as a function of true longitude for each of the individual solutions (1 to 10) are shown in Figure 4. Residuals for the combined fit to all the observations (11) are shown as a function of time in Figure 5, and as a function of true longitude, windowed for each individual sequence in Figure 6. For comparison, $O-C$ residuals as a function of true longitude are also provided relative to the model of Albers et al. (2012) in Figure 7. The rms residual for the entire ISS data set with respect to the model of Albers et al. (2012) is $9.1 \mathrm{~km}$, which compares favorably with the value of $8.2 \mathrm{~km}$ obtained for the actual fit to the ISS data (combined fit 11). By comparison, Albers et al. (2012) obtained an rms of $24.0 \mathrm{~km}$ with respect to their model, when fitting occultation data.

The relatively short time span of each image sequence (less than one orbital period of the ring) meant that the apsidal and 


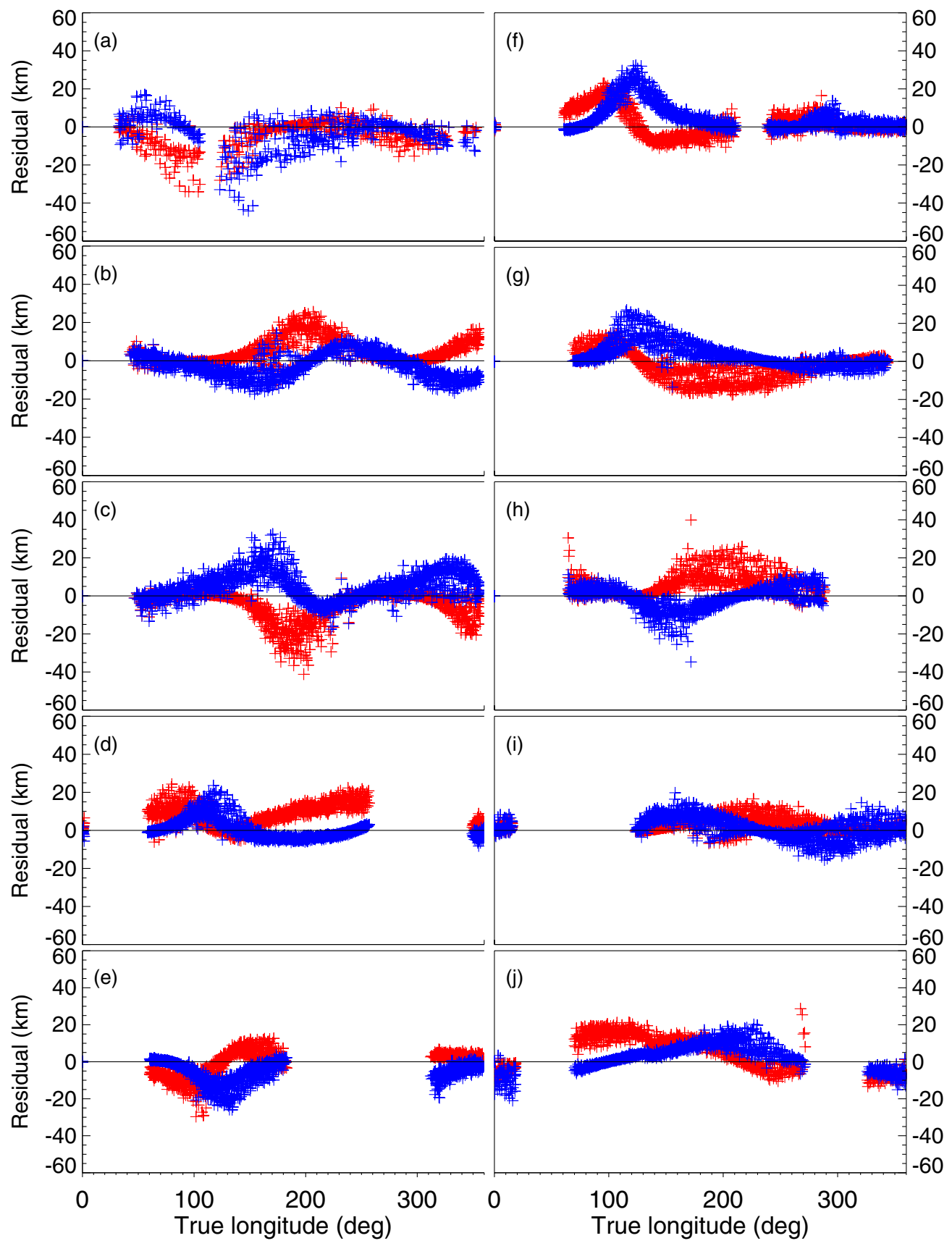

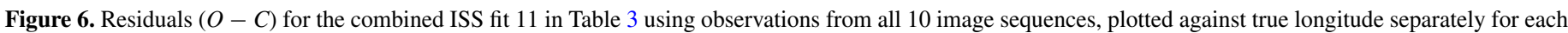

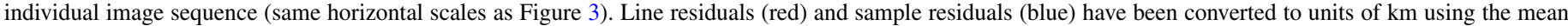
resolution for each image sequence. The plotted data are otherwise identical to that shown in Figure 5.

(A color version of this figure is available in the online journal.)

nodal rates of change due to the oblateness of Saturn were poorly constrained in the individual fits. These rates were therefore computed from the fitted semi-major axis values using the constants given in Table 2. In the case of the combined fits (11 and 12), with a time span of almost 3 years, good estimates of the apsidal precession rate were possible, with uncertainties comparable with the published stellar occultation values of Albers et al. (2012). However it was not possible to constrain the nodal rate satisfactorily and a fixed value has been used for this. In the ISS fits in general, the larger uncertainties in the fitted inclination and longitude of ascending node values compared with the eccentricity and longitude of periapse are most likely a consequence of viewing the ring from above the ring plane.
Using Equation (1) from Albers et al. (2012), we estimate that the maximum sensitivity of the observations to inclination varies from $\sim 11 \mathrm{~km}$ (rev 033) to $\sim 34 \mathrm{~km}$ (rev 088). This contrasts with sensitivities of $200-450 \mathrm{~km}$ for some occultation observations (Albers et al. 2012).

\section{DISCUSSION}

\subsection{Combined Orbital Solutions Using All Image Sequences}

Firstly, we consider fit 11 in Table 3, labeled "Combined ISS." Comparing this with the Albers et al. (2012) and Bosh et al. (2002) values, labeled 13 and 14 in Table 3, the semimajor axes and eccentricities are largely consistent within the 
Orbital Solutions for the Core of Saturn's F Ring

\begin{tabular}{|c|c|c|c|c|c|c|c|c|c|c|}
\hline Fit & $r e v^{\mathrm{a}}$ & $\begin{array}{l}\text { Epoch } \\
\text { (UTC) }\end{array}$ & $\begin{array}{c}\text { Semi-major } \\
\text { Axis } \\
(\mathrm{km})\end{array}$ & $\begin{array}{l}\text { Eccentricity } \\
\qquad\left(\times 10^{3}\right)\end{array}$ & $\begin{array}{l}\text { Long. of } \\
\text { Periapse } \\
\text { (deg) }\end{array}$ & $\begin{array}{c}\text { Apsidal } \\
\text { Precession Rate } \\
\left(\operatorname{deg}_{\text {day }}-1\right)\end{array}$ & $\begin{array}{l}\text { Inclination } \\
\left(\operatorname{deg} \times 10^{3}\right)\end{array}$ & $\begin{array}{l}\text { Long. of } \\
\text { Ascending Node } \\
\text { (deg) }\end{array}$ & $\begin{array}{c}\text { Nodal } \\
\text { Regression Rate } \\
\left(\operatorname{deg}_{\text {day }}{ }^{-1}\right)\end{array}$ & $\begin{array}{l}\mathrm{rms} \\
(\mathrm{km})\end{array}$ \\
\hline 1 & 033 & 2006 Nov 23 17:00:00.0 & $140232.9 \pm 0.4$ & $2.472 \pm 0.002$ & $339.94 \pm 0.03$ & $2.6977^{\mathrm{b}}$ & $4.5 \pm 0.2$ & $67 \pm 3$ & $-2.6853^{\mathrm{b}}$ & 5.5 \\
\hline 2 & 043 & 2007 Apr 20 07:20:00.0 & $140224.6 \pm 0.2$ & $2.4651 \pm 0.0005$ & $19.64 \pm 0.02$ & $2.6983^{\mathrm{b}}$ & $5.62 \pm 0.09$ & $25.3 \pm 0.9$ & $-2.6858^{b}$ & 3.3 \\
\hline 3 & 044 & 2007 May 6 06:00:00.0 & $140218.6 \pm 0.2$ & $2.3460 \pm 0.0008$ & $66.88 \pm 0.02$ & $2.6987^{\mathrm{b}}$ & $6.82 \pm 0.08$ & $358.9 \pm 0.7$ & $-2.6863^{\mathrm{b}}$ & 3.8 \\
\hline 4 & 080 & 2008 Aug 9 11:58:54.81 & $140220.8 \pm 0.1$ & $2.184 \pm 0.001$ & $231.62 \pm 0.02$ & $2.6986^{\mathrm{b}}$ & $6.15 \pm 0.06$ & $205.8 \pm 0.6$ & $-2.6861^{b}$ & 2.6 \\
\hline 5 & 088 & 2008 Oct $621: 45: 00$ & $140216.1 \pm 0.2$ & $2.312 \pm 0.001$ & $25.06 \pm 0.02$ & $2.6989^{\mathrm{b}}$ & $4.98 \pm 0.06$ & $40 \pm 1$ & $-2.6864^{\mathrm{b}}$ & 3.0 \\
\hline 6 & 091 & 2008 Oct $2820: 17: 00$ & $140213.36 \pm 0.09$ & $2.4725 \pm 0.0004$ & $89.00 \pm 0.02$ & $2.6991^{\mathrm{b}}$ & $5.28 \pm 0.05$ & $339.7 \pm 0.5$ & $-2.6866^{\mathrm{b}}$ & 2.3 \\
\hline 7 & 102 & 2009 Jan 30 09:45:00 & $140215.1 \pm 0.2$ & $2.3101 \pm 0.0008$ & $341.98 \pm 0.02$ & $2.6989^{\mathrm{b}}$ & $6.3 \pm 0.1$ & $102.3 \pm 0.7$ & $-2.6865^{b}$ & 3.5 \\
\hline 8 & 103 & 2009 Feb 9 16:14:00 & $140228.5 \pm 0.2$ & $2.429 \pm 0.001$ & $9.98 \pm 0.02$ & $2.6980^{\mathrm{b}}$ & $4.3 \pm 0.2$ & $116 \pm 2$ & $-2.6856^{\mathrm{b}}$ & 3.9 \\
\hline 9 & 109 & 2009 Apr 24 10:00:00 & $140222.5 \pm 0.2$ & $2.3066 \pm 0.0007$ & $209.19 \pm 0.02$ & $2.6984^{\mathrm{b}}$ & $2.8 \pm 0.1$ & $242 \pm 3$ & $-2.6860^{\mathrm{b}}$ & 3.4 \\
\hline 10 & 115 & 2009 Jul 28 18:35:00 & $140211.2 \pm 0.1$ & $2.4333 \pm 0.0007$ & $107.35 \pm 0.01$ & $2.6992^{\mathrm{b}}$ & $5.06 \pm 0.06$ & $313.7 \pm 0.8$ & $-2.6868^{\mathrm{b}}$ & 2.7 \\
\hline 11 & Combined ISS $^{\mathrm{c}}$ & $\mathrm{J} 2000$ & $140223.92 \pm 0.09$ & $2.3636 \pm 0.0004$ & $8.8 \pm 0.3$ & $2.7052 \pm 0.0002$ & $5.68 \pm 0.05$ & $5.3 \pm 0.6$ & $-2.6859^{b}$ & 8.2 \\
\hline 12 & Combined ISS $^{\mathrm{d}}$ & $\mathrm{J} 2000$ & $140220.8 \pm 0.3$ & $2.399 \pm 0.002$ & $16.7 \pm 0.9$ & $2.7028 \pm 0.0004$ & $5.1 \pm 0.1$ & $7 \pm 2$ & $-2.6861^{b}$ & 8.2 \\
\hline 13 & Albers et al. $(2012)^{\mathrm{e}}$ & $\mathrm{J} 2000$ & $140221.3 \pm 1.8$ & $2.35 \pm 0.02$ & $24.2 \pm 0.8$ & $2.70025 \pm 0.00029$ & $6.43 \pm 0.15$ & $15.0 \pm 1.4$ & -2.68778 & 24.0 \\
\hline 14 & Bosh et al. $(2002)^{\mathrm{f}}$ & $\mathrm{J} 2000$ & $140223.7 \pm 2.0$ & $2.54 \pm 0.05$ & $24.1 \pm 1.6$ & $2.7001 \pm 0.0004$ & $6.5 \pm 0.7$ & $16.1 \pm 3.6$ & -2.6876 & 5.7 \\
\hline
\end{tabular}

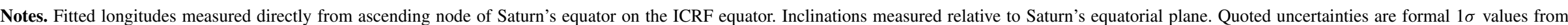
the fits.

a Full observation sequence names are ISS_revRF_FRSTRCHAN001_PRIME where rev is the number given in Column 2.

${ }^{\mathrm{b}}$ Calculated from semi-major axis.

${ }^{\mathrm{c}}$ Using all data points from all image sequences.

${ }^{\mathrm{d}}$ Using all image sequences, but restricted to data points $2^{\circ}$ or more ahead of Prometheus.

e Fit 2 from Albers et al. (2012, Table 3).

${ }^{\mathrm{f}}$ Fit 3 from Bosh et al. (2002, Table III) 


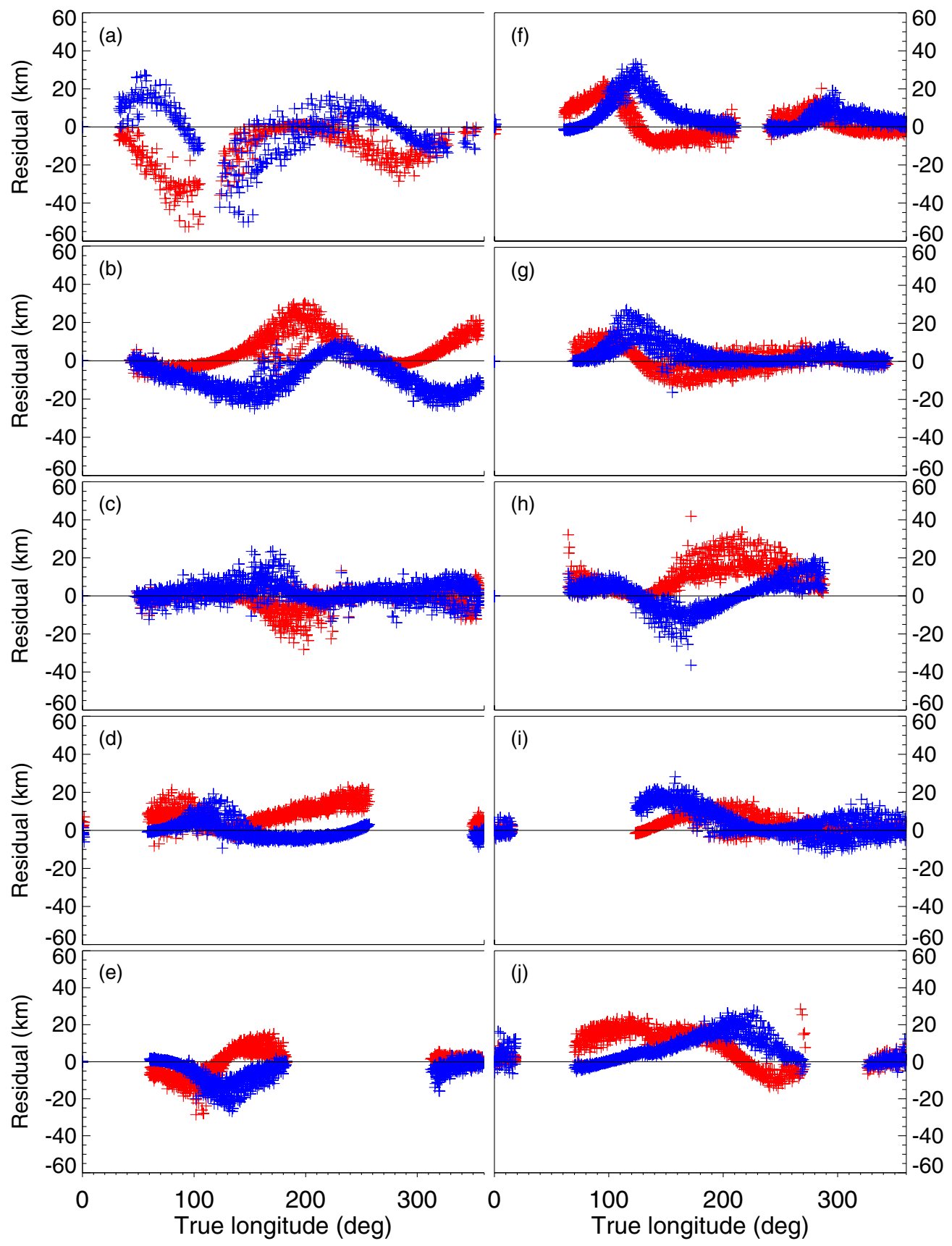

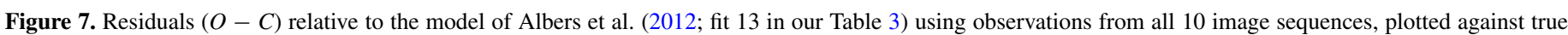

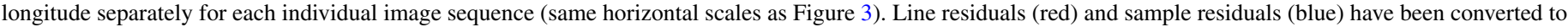
units of $\mathrm{km}$ using the mean resolution for each image sequence.

(A color version of this figure is available in the online journal.)

error bars, which are in general significantly smaller for the ISS solutions (by up to an order of magnitude in the case of the semi-major axis), reflecting the larger ISS observation data set (9805 pairs of observations). However, the periapse and apsidal rate from fit 11, and to a lesser degree the inclination and node, show a significant systematic shift relative to the Bosh et al. (2002) and Albers et al. (2012) values.

In searching for the source of this anomaly, one obvious possibility was a biasing effect caused by the close proximity of the satellite Prometheus to the ring in all the STREAMER/ CHANNEL images used in this study. We believe this possibility is supported by a further series of solutions using all image sequences but restricted subsets of the observations based on their longitudinal offset from Prometheus. The results are shown graphically in Figures 8 and 9. In both figures, the horizontal axis represents the number of degrees in longitude, $\theta$, ahead or behind Prometheus for observations included in a given fit. The figures differ in the way in which the input data were selected prior to fitting. In Figure 8, parts of the ring closest to Prometheus were progressively excluded, so that for example all plotted points with $\theta=2.0$ represent the fitted orbital elements for the case where only those parts of the ring more than $2^{\circ}$ ahead of Prometheus have been included in the fit, while for points with $\theta=-2.0$, only parts of the ring more than 2.0 behind Prometheus have been included. In Figure 9, each plotted point represents a fit to observations within a narrow range of angular 

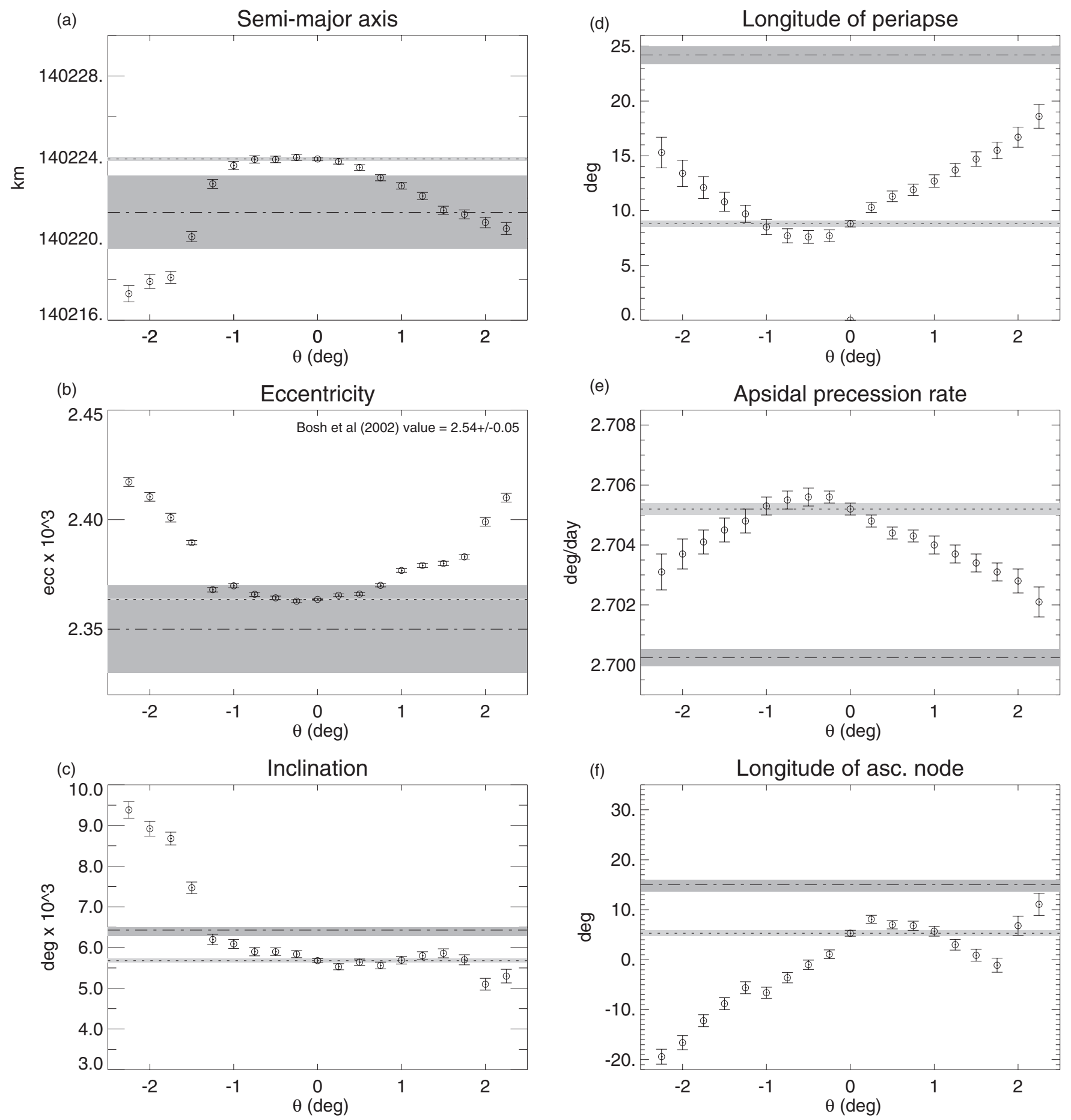

Figure 8. Fitted parameters for various combined fits, using all image sequences but progressively excluding data points corresponding to parts of the ring $<|\theta|^{\circ}$ in longitude away from Prometheus. Error bars are $1 \sigma$ uncertainties. Dotted lines correspond to the combined fit using all data points (fit 11). Dot-dashed lines correspond to the Albers et al. (2012) values, with uncertainties shown by the shaded areas. In (b) the Bosh et al. (2002) eccentricity value, which is off-scale, is stated numerically. Values at 2.0 correspond to fit 12 , Table 3. Plot scales are the same as for Figure 9.

offset from Prometheus, 0.25 wide, centered on the plotted point. In both figures, the horizontal dotted lines represent the values from the combined fit 11 (which uses all data points) and the dot-dashed lines those from Albers et al. (2012), with their uncertainties shown by the gray shading. In Figure 8, the points at $\theta=0^{\circ}$ are from combined fit 11 , using all data points.

Moving to the extreme right or left on the horizontal axis in Figures 8 and 9, the solutions therefore represent parts of the ring that are progressively more distant from Prometheus, and we see that in the case of Figures 8(a) the semi-major axis, (d) the longitude of periapse and (e) the apsidal precession rate, there is a trend away from the combined ISS fit 11 toward the Albers et al. (2012) solution, labeled 13 in Table 3. The suggestion from these results is therefore that the bias in combined fit 11 is caused by the proximity of Prometheus in the images used in this study, demonstrating also that the effects of a given encounter are felt by parts of the ring several degrees of orbital longitude away from Prometheus, in either direction. The results shown in Figure 9 are noisier, due to the smaller subset of observations used for each fit (also reflected in the larger error bars) and hence 

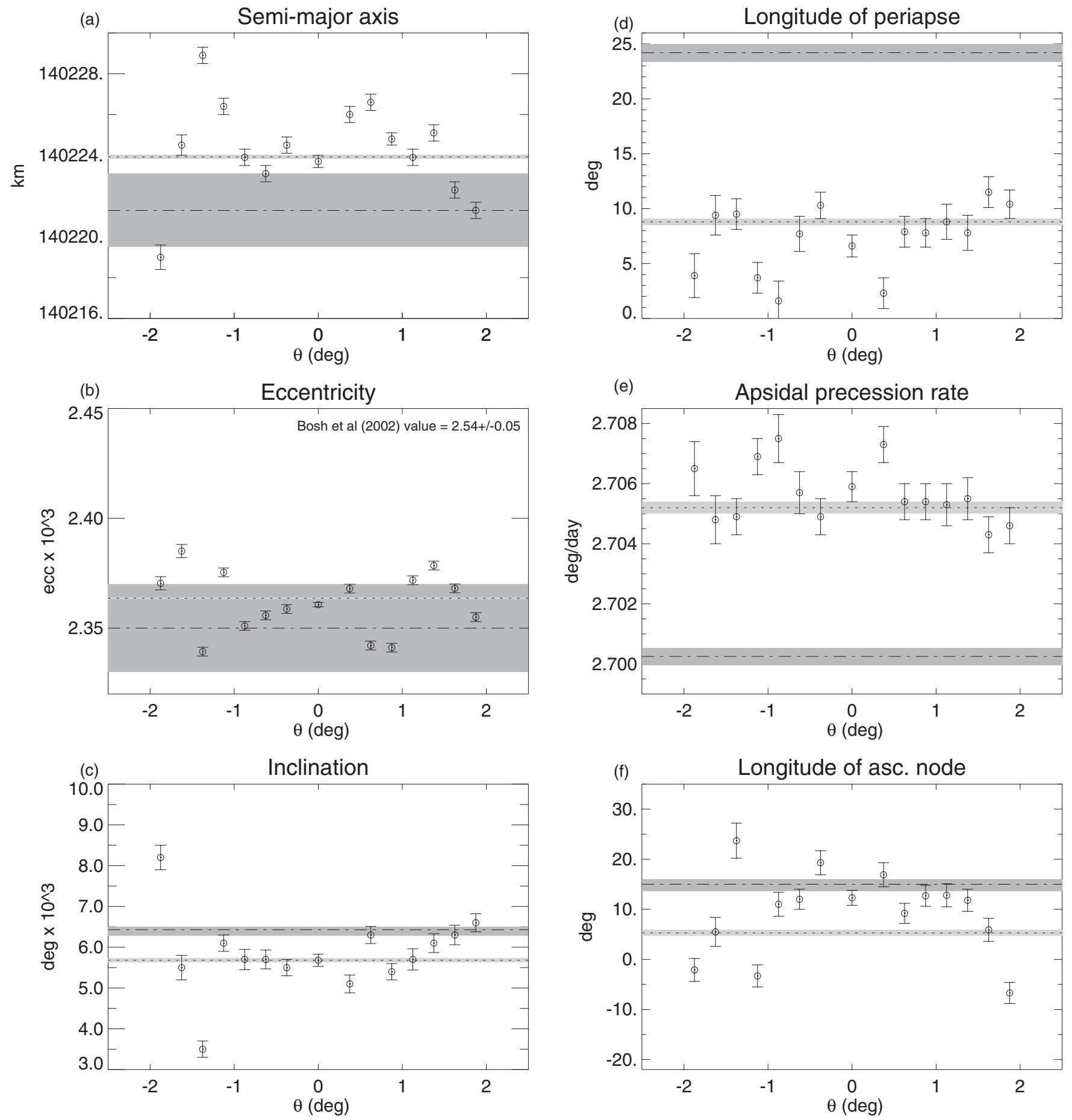

Figure 9. Fitted parameters for various combined fits, using all image sequences but for different restricted ranges of longitudinal distance, $\theta^{\circ}$, from Prometheus. The restricted ranges are 0.25 wide, centered on each plotted point. Error bars are $1 \sigma$ uncertainties. Dotted lines correspond to the combined fit using all data points (fit 11). Dot-dashed lines correspond to the Albers et al. (2012) values, with uncertainties shown by the shaded areas. In (b) the Bosh et al. (2002) eccentricity value, which is off-scale, is stated numerically. Plot scales are the same as for Figure 8.

any possible trends are less convincing. However, we believe the suggestion of a similar trend can be seen in the semi-major axis (Figure 9(a)).

The inclination values, Figure 8(c), appear to diverge strongly from both the combined fit 11 and Albers et al. (2012) values in the negative $\theta$ direction, i.e., after Prometheus has recently passed this section of ring. However, for positive $\theta$, at least for the range of $\theta$ considered, the values stay closer to the combined fit 11 values. The same is true of the fitted node values in Figure 8(f), although there is more of a suggestion of convergence toward the Albers et al. (2012) value for positive $\theta$. We also note that in the case of the eccentricity, there is an inconsistency between the Bosh et al. (2002) and Albers et al. (2012) values (see Table 3). Albers et al. (2012) account for this by suggesting that the smaller Bosh et al. (2002) data set is less sensitive to eccentricity and precession rate, however we find that the eccentricity values from the combined ISS fits converge toward the Bosh et al. (2002) value rather than the Albers et al. (2012) value (Figure 8(b)). The full set of fitted elements for the $2^{\circ}$ case corresponds to fit 12 in Table 3 for comparison 

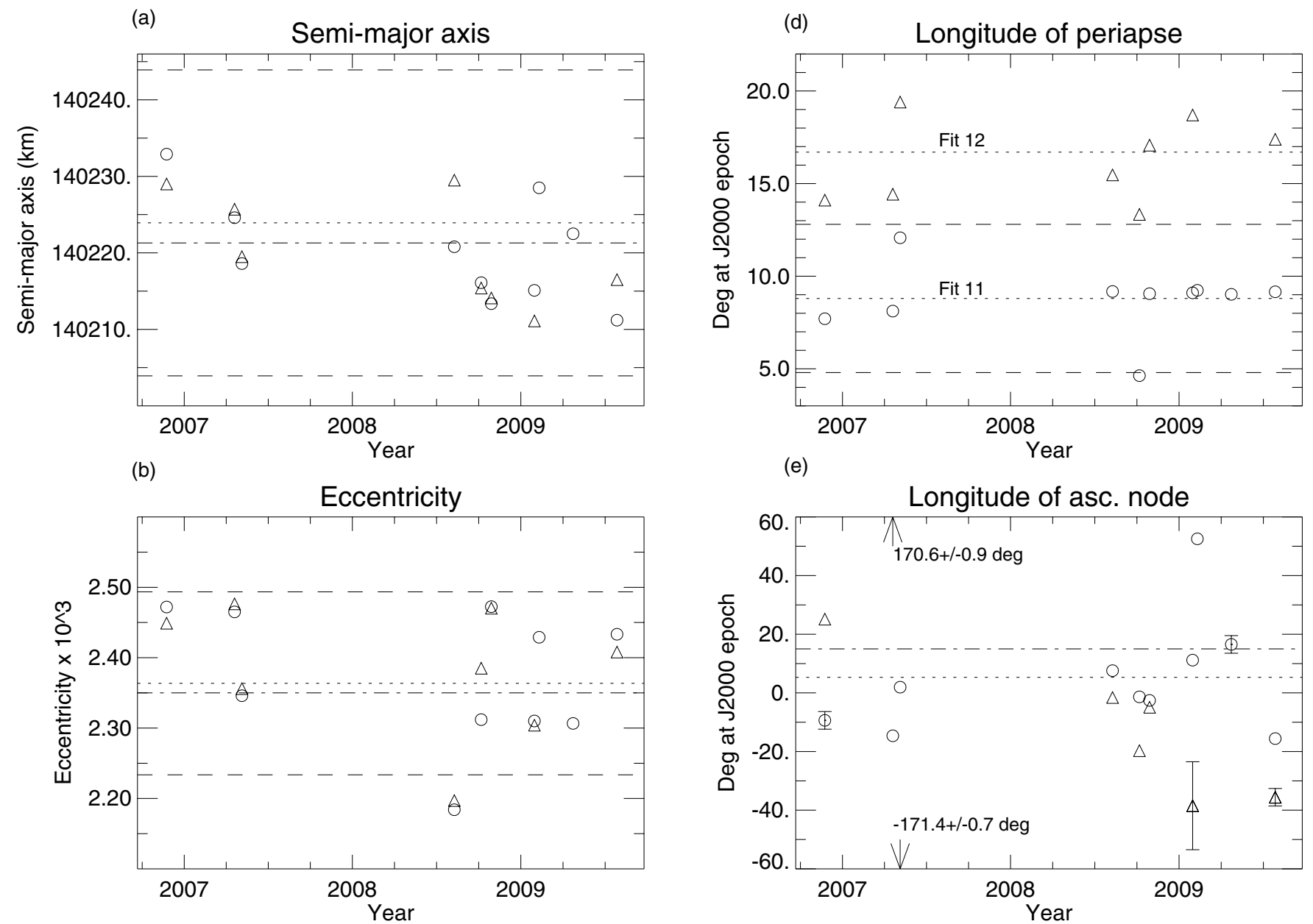

(c)

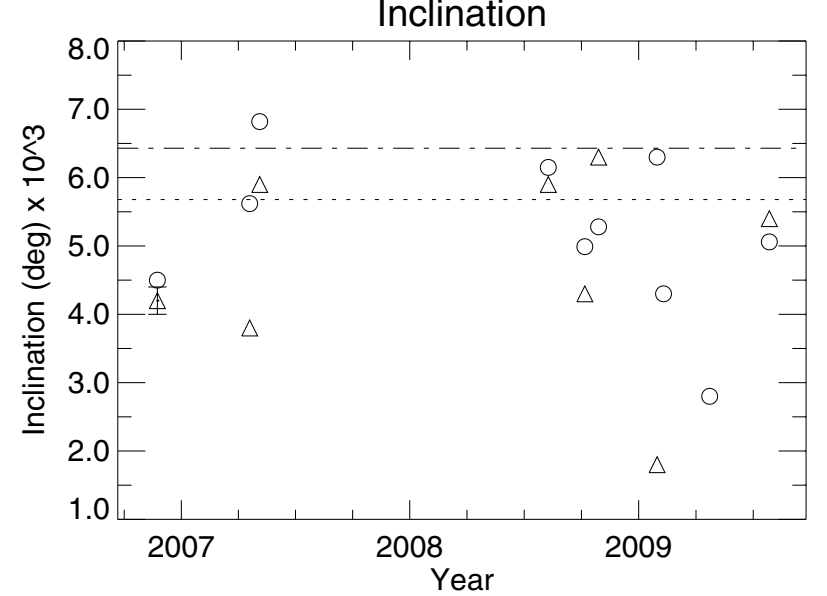

Figure 10. Fits 1-10 vs. time. Uncertainties \&symbol size, except where error bars shown. Dotted lines: combined fit 11, dot-dashed lines: Albers et al. (2012). Triangles: fits excluding parts of the ring $<2^{\circ}$ ahead of Prometheus. (a) Semi-major axis. Outer dashed lines $\pm 20 \mathrm{~km}$ either side of fit 11 value. (b) Eccentricity. Outer dashed lines $\pm 13 \times 10^{-5}$ either side of fit 11 value. (c) Inclination. (d) Circles: longitude of periapse precessed back to J2000 epoch using fit 11 rate. Dashed lines $\pm 4^{\circ}$ either side of fit 11 value. Triangles: fits excluding parts of the ring $<2^{\circ}$ ahead of Prometheus, precessed back to J2000 using combined-fit 12 rate. Albers et al. (2012) value is $24.2 \pm 0.8$ (off scale). (e) Longitude of ascending node precessed back to J2000 using fit 11 rate.

with fit 11, which uses all data points. Again, comparable trends in Figure 9 are less convincing, possibly reflecting the larger uncertainties associated with the smaller size of the data sets contributing to each fitted point.

\subsection{Orbital Solutions for Individual Image Sequences}

The much larger residuals shown in both Figures 6 and 7 compared to Figure 4 clearly show the extent to which the averaged orbits represented by combined fit 11 and the model of Albers et al. (2012) depart from the best local solutions, 1-10 in Table 3.

The fitted parameters for the individual solutions, 1-10, are plotted at their respective time epochs in Figure 10. In this figure, the corresponding values from the combined fit 11 are given by the horizontal dotted lines, while the Albers et al. (2012) values are represented by the dot-dashed lines. In addition to the 
individual solutions, $1-10$ in Table 3 , an alternative solution has been produced for each individual image sequence excluding parts of the ring less than $2^{\circ}$ ahead of Prometheus, except for the rev103 and 109 sequences, for which convergence could not be achieved due to the limited number of observations. These values are not listed in Table 3 (fit 12 is the corresponding combined fit), but are represented by the triangular symbols in Figure 10.

Differential precession anti-aligns the periapses of Prometheus and the F ring approximately every 19 years, with the latest anti-alignment having occurred in 2009 December. The images in this study cover a period of three years leading up to 2009 July, just before the most recent anti-alignment. These image sequences therefore sample the ring at a time when the magnitude of the perturbations from Prometheus are approaching their maximum value. The maximum absolute changes in semi-major axis, eccentricity, and longitude of periapse at antialignment are $\sim 19 \mathrm{~km}, 13 \times 10^{-5}$ and $4^{\circ}$, respectively (Murray et al. 2008). Since Murray et al. (2008) considered an equatorial ring, they do not quote values for inclination and node. These expected changes in semi-major axis, eccentricity, and periapse at anti-alignment with respect to combined fit 11 are marked by the outer horizontal dashed lines in Figures 10(a), (b), and (d) and referring to these figures, we see that the observed variations in these elements given by the circular symbols are within the expected limits, except for one point in eccentricity, which is marginally outside its lower limit. The triangular symbols show a similar scatter and, unlike the averaged solutions (Figure 8), do not show a trend toward the Albers et al. (2012) values, indicating that for the particular segments of the F ring imaged by these observations, the local effect of the latest Prometheus encounter is more significant than the superposition of past encounters.

The periapse values in Figure 10(d) were obtained by precessing the fitted values in Table 3 (fits 1-10) back to the J2000 epoch, using the precession rate from combined fit 11 of 2.7052 day $^{-1}$. For comparison, a horizontal dotted line is shown in Figure 10(d), corresponding to the combined fit 11 value at the J2000 epoch, of 8.8. Thus we see from Figure 10(d) that the individual fitted periapses for solutions 1-10 are consistent with the fitted periapse and precession rate from combined fit 11 , with the expected superimposed scatter of $\sim \pm 4^{\circ}$ (see above) around the combined fit value. We recall also that the combined fit 11 periapse of $8.8 \pm 0.3$ is significantly different from the Albers et al. (2012) value of $24.2 \pm 0.8$. When excluding parts of the ring that are less than $2^{\circ}$ ahead of Prometheus, given by the triangular values in Figure 10(d), the fitted values are now consistent with the corresponding combined fit 12 solution, as expected, shown by the horizontal dotted line corresponding to $16.7 \pm 0.9$. Thus we see that, unlike the semi-major axis, eccentricity, and inclination, the local periapse values behave like the averaged solution, shifting toward the Albers et al. (2012) value when the effects of the latest Prometheus encounter are excluded from the fit.

The two different methods of data selection described in the previous section for the combined fits (Figures 8 and 9) were also compared on two individual image sequences, ISS_043RF_FRSTRCHAN001_PRIME and ISS_115RF_FRSTRCHAN001_PRIME. Although not reproduced here, we note that the results also showed a variability away from the $0^{\circ}$ position, which we believe could be due to the local perturbing effects of Prometheus (the variability in the semi-major axis is of the same order of magnitude as for the combined-fit results). However, the shapes of the trends were different for each sequence and also different from the combined fit results. Also, unlike the combined fits, the results did not show a trend toward the Albers et al. (2012) values. We argue (next paragraph) that this simply reflects the local variability of the ring, since each individual image sequence samples a relatively small range of co-rotating longitude.

In general, we maintain that the local variability ("scatter") in the orbits obtained from the individual image sequences, apparent in Figure 10, can be understood in the context of Figure 1: since a different segment of the ring with a different history has been imaged in each image sequence, each individual solution represents a model of the orbit of that particular $\sim 5^{\circ}$ segment of the ring. For example, in Figure 1, the "piece" of the $\mathrm{F}$ ring imaged in the STREAMER/CHANNEL sequence shown is marked by the vertical lines on the FMOVIE display. If a different section of ring had been sampled, a different orbital solution would have resulted, reflecting the local variability visible in the FMOVIE mosaic. For comparison, the particular fixed window in true longitude through which the entire ring was viewed in the FMOVIE is shown by the vertical lines in the STREAMER/CHANNEL display. In contrast, Figure 10(d) shows clearly that the fitted periapse values for each image sequence, although scattered, behave somewhat differently from the fitted semi-major axis, eccentricity, inclination, and node values. As discussed above, the local fitted periapse values are consistent with the combined fit values (fits 11 and 12), which essentially represent the averaged orbit over the period 2006 November to 2009 July.

\subsection{Apsidal Precession}

We have already noted that the observed periapses for the individual segments of the ring imaged by the STREAMER/ CHANNEL movies are locked to the averaged orbit of the ring as a whole. In addition, based on published values for the mean motion and precession rate of the F ring and the mean motion of Prometheus, we note that the ratio between the synodic period for Prometheus and the F ring, and the precession rate of the F ring is $1.966 \pm 0.004$. Hence, an approximate empirical commensurability exists such that $n_{\text {Prom }}-n_{\text {Fring }} \approx 2 \dot{\varpi}_{\text {Fring }}$, where $\dot{\varpi}$ is the apsidal precession rate of the F ring and $n_{\text {Prom }}$ and $n_{\text {Fring }}$ are the mean motions of Prometheus and the F ring, respectively.

To obtain the value of the ratio $1.966 \pm 0.004$, we have used the Albers et al. (2012) values of the precession rate of the F ring, $2.70025 \pm 0.00029 \mathrm{day}^{-1}$ and the mean motion of the F ring, $581.979 \pm 0.011 \mathrm{day}^{-1}$, and the French et al. (2003) estimate of the mean motion of Prometheus of 587.28747 $\pm 0.00005 \mathrm{day}^{-1}$, including their respective uncertainties. However, given that the mean motion of Prometheus changes approximately every 6.2 years after close approach with Pandora (French et al. 2003; Goldreich \& Rappaport 2003) and also given the results of this work, which show that the mean motion of the $F$ ring is locally variable, the ratio may vary by significantly more than the quoted uncertainty, and there may be parts of the F ring where, locally, an exact commensurability exists. We hesitate to refer to this as a resonance since the equivalent argument in longitude, $\phi=\lambda_{\text {Prom }}-\lambda_{\text {Fring }}-2 \varpi_{\text {Fring }}$ does not satisfy the D'Alembert rules. However, this is an empirical relationship that does not rule out the existence of other terms: they may simply be too small to detect (see, for example, Duriez \& Vienne 1997 and Lainey et al. 2006).

The dynamical significance of this relationship is currently unclear, but its nature is such that a ring particle that is at periapse (apoapse) when it encounters Prometheus, will be at 
apoapse (periapse) the next time it encounters Prometheus, and so on. It follows that it may be possible for parts of the ring at particular orbital phases, for example at quadrature, to have encounters that effectively cancel out, thereby affecting the time scale for their orbital evolution. This in turn implies that, through this mechanism, different parts of the ring can have a different evolution. However this requires further investigation.

\section{CONCLUSIONS}

Using images originally designed to track the effects of Prometheus on Saturn's F ring, we have been able to detect significant local variability in the orbit of the ring consistent with its complex visual appearance. Comparing individual local orbits as a function of time, the orbital elements show a scatter about the values obtained from a combined fit over all observations, reflecting the different local history of the segment of ring sampled by a given image sequence. Values are scattered within the expected limits due to a single Prometheus perturbation ( $\pm \sim 19 \mathrm{~km}$ in semi-major axis), suggesting that in the parts of the ring studied here, the effects of repeated encounters do not constructively accumulate beyond these limits. However this may not hold in general and does not rule out the possibility of orbital evolution in other parts of the ring. Minimizing the effects of the latest Prometheus perturbation still results in a scatter about the averaged values.

While similarly scattered between expected limits, local periapse values are consistent with the averaged orbit, and converge toward the Albers et al. (2012) result when the effects of the latest Prometheus perturbation are excluded. Thus, while in a "piecewise" sense each local ring segment has its own orbit reflecting the local evolution of that part of the ring, the local periapses are locked to the averaged orbit and reflect a dynamical property of the ring as a whole, i.e., despite the local variability, our results are consistent with the observed fact that the F ring precesses uniformly, presumably due to self-gravity and/or collisional effects.

A combined orbital solution using all the image sequences over the full time span of the observations, 2006 November to 2009 July, represents an averaged orbit over that timespan, consistent with orbits derived from stellar occultations by Bosh et al. (2002) and Albers et al. (2012) except for a small systematic bias ( $\sim 7 \mathrm{~km}$ in semi-major axis) arising from the proximity of Prometheus in the images used in this study.

Finally, we note the existence of an approximate empirical commensurability such that $n_{\text {Prom }}-n_{\text {Fring }} \approx 2 \dot{\varpi}_{\text {Fring }}$, where $\dot{\varpi}$ is the apsidal precession rate of the $\mathrm{F}$ ring and $n_{\text {Prom }}$ and $n_{\text {Fring }}$ are the mean motions of Prometheus and the F ring, respectively. The nature of the relationship suggests that a ring particle which is at periapse (apoapse) at encounter with Prometheus, will again be at apoapse (periapse) at the next encounter, while particles at quadrature may experience perturbations that effectively cancel out. However, this requires further investigation.

This study has been opportunistic in the sense that it has made use of images originally designed for a different purpose, which have nevertheless proved useful for determining the orbit of the ring. To augment this work, further observation sequences have already been designed with the aim of tracking parts of the ring away from the local perturbing effects of Prometheus. We also anticipate using the current methodology to analyze the local orbital characteristics of strands of material emerging from the core of the ring.

The authors thank the members and associates of the Cassini ISS team, particularly Mike Evans and Kevin Beurle for designing the image sequences used in this work. Thanks to Nick Attree and Valery Lainey and also to the anonymous reviewer for helpful insights and comments. This work was supported by the Science and Technology Facilities Council (grant number $\mathrm{ST} / \mathrm{F} 007566 / 1)$.

\section{REFERENCES}

Acton, C. H. 1996, P\&SS, 44, 65

Albers, N., Sremcevic, M., Colwell, J. E., \& Esposito, L. W. 2012, Icar, 217,367

Attree, N., Murray, C. D., Cooper, N. J., \& Williams, G. A. 2012, ApJL, 755, L27

Bosh, A. S., Olkin, C. B., French, R. G., \& Nicholson, P. D. 2002, Icar, 157,57

Charnoz, S., Porco, C. C., Deau, E., et al. 2005, Sci, 310, 1300

Duriez, L., \& Vienne, A. 1997, A\&A, 324, 366

French, R. G., McGhee, C. A., Dones, L., \& Lissauer, J. J. 2003, Icar, 162, 143

Giuliatti Winter, S. M. 1994, PhD thesis, Univ. London

Goldreich, P., \& Rappaport, N. 2003, Icar, 162, 391

Høg, E., Fabricius, C., Makarov, V. V., et al. 2000, A\&A, 355, L27

Jacobson, R. A. 2004, AJ, 128, 492

Kliore, A. J., Patel, I. R., Lindal, G. F., et al. 1980, JGR, 85, 5857

Lainey, V., Duriez, L., \& Vienne, A. 2006, A\&A, 456, 783

Lawson, C. L., \& Hanson, R. J. 1975, Solving Least Squares Problems (Philadelphia, PA: SIAM)

Murray, C. D., Beurle, K., Cooper, N. J., et al. 2008, Natur, 453, 739

Murray, C. D., Chavez, C., Beurle, K., et al. 2005, Natur, 437, 1326

Showalter, M. R. 2004, Icar, 171, 356

Zacharias, N., Urban, S. E., Zacharias, M. I., et al. 2004, AJ, 127, 3043 